Pacific Journal of Mathematics

GTON SPACE TOPOLLGGirs 


\title{
FUNCTION SPACE TOPOLOGIES
}

\author{
J. D. HANSARD
}

S. Naimpally [3] introduced the graph topology, $I$, for function spaces. H. Poppe [5] showed that if the graph topology is finer than the topology of uniform convergence, $\tau_{u}$, or finer than the finest of the $\sigma$-topologies of Arens and Dugundji, $\tau$, and if the range space is the real line, $R$, then the domain is countably compact.

We assume our range space is $R$ and that our domain space $X$ is $T_{1}$. In most of this paper we deal with topologies on $C(X)$ the set of continuous real-valued functions on $X$. We show that $\Gamma=\tau=\tau_{u}$ on $C(X)$ if and only if $X$ is countably compact. Further, we find that when $X$ is locally connected, $\tau_{u} \subset \tau$ on $C(X)$ if and only if $X$ has finitely many components.

In order to determine conditions under which $\tau \subset \tau_{u}$, we introduce a map extension property between complete regularity and normality and show that for domain spaces $X$ having this property, $\tau \subset \tau_{u}$ on $C(X)$ if and only if $X$ is countably compact. We indicate further applications of this map extension property and compare it to weak normality.

We let $Y^{X}$ denote the set of all functions from $X$ to $Y$. For $f \in Y^{X}$, let $G(f)=\{(x, f(x)): x \in X\}$, and for $U \subset X \times Y$, let $F_{U}=$ $\left\{f \in Y^{X}: G(f) \subset U\right\}$. If $K \subset X$ and $U \subset Y$, define $(K, U)=\left\{f \in Y^{X}\right.$ : $f(K) \subset U\}$. For $\varepsilon>0$, let $V_{\varepsilon}(f)=\left\{g \in R^{X}:|f(x)-g(x)|<\varepsilon\right.$ for all $\left.x \in X\right\}$. Also, define $N_{\varepsilon}(x)=\{y \in R:|y-x|<\varepsilon$, and for any set $K$, let $c K$ be the complement of $K$.

The graph topology, defined in [3], has a basis consisting of the sets of the form $F_{U}$ where $U$ is open in $X \times Y$. The finest of the $\sigma$-topologies, defined in [1], has a subbasis consisting of the collection of all sets of the form $(K, U)$ where $K \subset X$ is closed and $U \subset Y$ is open. The topology of uniform convergence has a basis consisting of all sets of the form $V_{\varepsilon}(f)$ where $f \in R^{X}$ and $\varepsilon>0$.

1. Two lemmas. The first of our lemmas is a characterization of $\tau$ which we find convenient to use throughout this paper. This result provides us with a basis for $\tau$. Because of the nature of these basic elements, the relation between $\tau$ and $\Gamma$ is immediately made clear, and we are able to think of $\tau$, intuitively, as a special kind of graph topology rather than as a set-open topology.

H. Poppe [5] showed that $\tau$ has a subbasis consisting of sets of the form $[K \times L]=\{f: G(f) \cap K \times L=\varnothing, K \subset X$ closed, $L \subset Y$ closed $\}$. Thus a basic open set of functions in $\tau, \bigcap_{1}^{n}\left[K_{i} \times L_{i}\right]$, is completely 
determined by the set of points $\bigcup_{1}^{n} K_{i} \times L_{i}$.

LEMMA 1. The topology $\tau$ has a basis consisting of sets of the form $F_{U}$ where $U=\bigcup_{i \in \mathscr{A}} A_{i} \times B_{i}$ and $A_{i} \subset X$ and $B_{i} \subset Y$ are open for each $i \in \mathscr{A}$ and $\mathscr{A}$ is finite.

Proof. Choose $[K \times L] \in \tau$. Clearly $[K \times L]=F_{V}$ where $V=$ $c K \times Y \cup X \times c L$, so that $[K \times L]$ is open in the topology generated by sets of the form $F_{U}$ described above. Now consider $F_{U}$ where $U=\bigcup_{i \in \mathscr{A}} A_{i} \times B_{i}, A_{i} \subset X$ and $B_{i} \subset Y$ are open for each $i \in \mathscr{A}$ and $\mathscr{A}$ is finite. In order to see that $c U=c\left(\mathbf{U}_{i \in \leftrightarrow} A_{i} \times B_{i}\right)=$ $\bigcup_{\mathscr{B} \subset \mathscr{A}}\left(c \bigcup_{i \notin \mathscr{B}} A_{i} \times c \bigcup_{i \in \mathscr{B}} B_{i}\right)$, choose $(x, y) \in c U$. Then for each $i$, either $x \notin A_{i}$ or $y \notin B_{i}$. If $\mathscr{B}<\mathscr{A}$ is chosen so that $i \in \mathscr{B}$ if and only if $x \in A_{i}$, then $(x, y) \in\left(c \bigcup_{i \notin \mathscr{B}} A_{i} \times c \bigcup_{i \in \mathscr{B}} B_{i}\right)$. If $(x, y) \in$

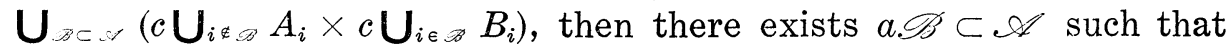
$(x, y) \in\left(c \bigcup_{i \notin \mathscr{B}} A_{i} \times c \bigcup_{i \in \mathscr{B}} B_{i}\right)$ implying that $x \notin A_{i}$ for $i \notin \mathscr{B}$ while $y \notin B_{i}$ for $i \in \mathscr{B}$. This establishes set equality and from this we see that

$$
F_{U}=\left[\bigcup_{\mathscr{B} \subseteq \mathscr{A}}\left(c \bigcup_{i \notin \mathscr{S}} A_{i} \times c \bigcup_{i \in \mathscr{B}} B_{i}\right)\right]=\bigcap_{\mathscr{A} \subset \mathscr{A}}\left[\left(c \bigcup_{i \notin \mathscr{B}} A_{i} \times c \bigcup_{i \in \mathscr{B}} B_{i}\right)\right]
$$

showing that $F_{U}$ is open in $\tau$.

Lemma 2. If $X$ is countably compact, then for $f \in C(X)$ and $G(f) \subset U$ where $U$ is open in $X \times R$, there exists an $\varepsilon>0$ such that $\{x\} \times N_{\varepsilon}(f(x)) \subset U$ for any $x \in X$.

Proof. If the condition fails, then for $\varepsilon=1,1 / 2,1 / 3, \ldots$ there exist points $x_{1}, x_{2}, x_{3}, \cdots$, respectively, such that $\left\{x_{n}\right\} \times N_{1 / n}\left(f\left(x_{n}\right)\right) \not \subset U$. Suppose $\left\{x_{n}\right\}$ clusters to the point $x$. Then $(x, f(x)) \in U$ and there exists an open set $V$ containing $x$ and an $\varepsilon>0$ such that $V \times$ $N_{\varepsilon}(f(x)) \subset U$. We can suppose $f(V) \subset N_{\varepsilon / 2}(f(x))$ using the continuity of $f$. Then choosing $n_{0}$ such that $1 / n_{0}<\varepsilon / 2$ and $m>n_{0}$ such that $x_{m} \in V$, we see that $\left\{x_{m}\right\} \times N_{1 / m}\left(f\left(x_{m}\right)\right) \subset V \times N_{\varepsilon}(f(x)) \subset U$ which is a contradiction.

We remark that the converse of this lemma is also true. This follows from the fact that if $X$ is not countably compact, then there exists a sequence, $\left\{x_{n}\right\}$, with no cluster points. Let $U$ be open and $G(f) \subset U$. Then let $V=U-\left[\bigcup_{1}^{\infty}\left\{x_{n}\right\} \times c N_{1 / n}\left(f\left(x_{n}\right)\right)\right]$. The set $V$ is open, contains the gragh of $f$, and no $\varepsilon>0$ exists satisfying the condition.

This lemma together with its converse states that the complement of an open set containing the graph of an element of $C(X)$ can get "close" to the graph of $f$ if and only if $X$ is countably compact. 


\section{Comparisons of $\Gamma$ with $\tau_{u}$ and $\tau$.}

Theorem 3. A space $X$ is countably compact if and only if $\Gamma=\tau_{u}$ on $C(X)$.

Proof. Poppe [5] showed that when $\Gamma \subset \tau_{u}$ on $C(X)$, then $X$ is countably compact. The converse of this result follows immediately from Lemma 2.

A proof analogous to Poppe's can be made with $R$ replaced by any first countable $T_{1}$ space containing a nonisolated point. Also, the proof of Lemma 2 above remains valid with $R$ replaced by any metric space. Hence, Theorem 3 is true for the case when $R$ is replaced by any metric space containing a nonisolated point.

We note that $\tau_{u} \subset \Gamma$ on $C(X)$ always holds since $\{(x, y):|y-f(x)|<\varepsilon\}$ is an open set for any $\varepsilon>0$ and for $f \in C(X)$. In fact, the function $f$ is continuous if and only if the set is open for every $\varepsilon>0$.

THeorem 4. A space $X$ is countably compact if and only if $\Gamma=\tau$ on $C(X)$.

Proof. Poppe [5] showed that when $\Gamma \subset \tau$ on $C(X)$, then $X$ is countably compact. It is clear from Lemma 1 that $\tau \subset \Gamma$. Therefore, we have only to show that when $X$ is countably compact, then $\Gamma \subset \tau$. Suppose $X$ is countably compact and choose $F_{U} \in \Gamma$ with $f \in F_{U}$. By Lemma 2, there exists and $\varepsilon>0$ such that $\{x\} \times N_{\varepsilon}(f(x)) \subset U$ for every $x \in X$. Let $\left\{W_{i}: 1=1,2, \cdots, n\right\}$ be an open cover of $f(X)$ such that the diameter of each $W_{i}$ is less than $\varepsilon$, and define $V=$ $\mathbf{U}_{1}^{n} f^{-1}\left(W_{i}\right) \times W_{i}$. Clearly, $F_{V} \in \tau$ and it is easy to show that $F_{V} \subset F_{U}$ by showing that $V \subset U$.

We remark that Theorem 4 can be established for the case when $R$ is replaced by any Lindelöf metric space containing a nonisolated point. We need the metric space property to use Lemma 2, the Lindelöf property to insure that $f(X)$ is compact when $X$ is countably compact, and the nonisolated point (together with first countable and $T_{1}$ ) for Poppe's proof.

When Poppe showed that $\Gamma \subset \tau$ on $C(X)$ implies that $X$ is countably compact, he remarked that if $X$ is completely regular and if $\Gamma \subset \tau$ on $C(X)$, then $X$ must be compact. Theorem 4 shows that his statement is incorrect. Poppe made a similar statement when comparing $\Gamma$ with $\tau_{u}$ and Theorem 3 indicates that it also is incorrect.

Naimpally [3] showed that when $X$ is a compact $T_{2}$ space, the 
graph topology and the compact-open topology coincide. This fact together with Theorem 4 shows that when $X$ is a compact $T_{2}$ space, the compact-open topology is the same as $\tau$ for $C(X)$ and so has basic open sets of the form $F_{U}$ where $U$ is expressible as a finite union of Cartesian products of open sets.

3. Comparisons of $\tau$ with $\tau_{u}$. S. Naimpally and C. Pareek ([4], Ex. 5.10) assert that $\tau_{u} \not \subset \tau$ on $C(X)$ when $X=R$. The following result shows that their assertion is wrong.

\section{LEMma 5. When $X$ is connected, $\tau_{u} \subset \tau$ on $C(X)$.}

Proof. Assume $X$ is connected and choose $V_{\varepsilon}(f) \in \tau_{u}$. Fix $x \in X$ and define $p_{k}=k(\varepsilon / 2)+f(x)$ for $k \in I$ where $I$ is the set of integers. Then define $B_{1}=N_{\varepsilon / 2}\left(p_{0}\right), B_{2}=\bigcup_{i \in I} N_{\varepsilon / 2}\left(p_{3 i+1}\right), \quad B_{3}=\mathbf{U}_{i \in I} N_{\varepsilon / 2}\left(p_{3 i+2}\right)$, $B_{4}=\bigcup_{i \neq 0} N_{\varepsilon / 2}\left(p_{3 i}\right)$, and $A_{i}=f^{-1}\left(B_{i}\right)$ for $i=1,2,3,4$. We assert that $f \in F_{W} \subset V_{\varepsilon}(f)$ where $W=\bigcup_{1}^{4} A_{i} \times B_{i}$.

In order to establish this assertion, notice that $U=$ $\mathrm{U}_{i \in I} f^{-1}\left(N_{\varepsilon / 2}\left(p_{i}\right)\right) \times N_{\varepsilon / 2}\left(p_{i}\right) \subset W$ and certainly $G(f) \subset U$, so that $f \in F_{w}$. If $g \in F_{w}$, then $G(g)$ is connected, and $G(g) \cap f^{-1}\left(N_{\varepsilon / 2}\left(p_{0}\right)\right) \times N_{\varepsilon / 2}\left(p_{0}\right) \neq \varnothing$ implying that $G(g) \cap U \neq \varnothing$. We will show that $W=U \cup(W-U)$ is a separation of $W$, so that $G(g) \subset U$. This will establish the fact that $F_{W} \subset F_{U}$ and surely $F_{U} \subset V_{\varepsilon}(f)$, which will complete the proof.

Now we show that $W=U \cup(W-U)$ is a separation. Call a set of the form $f^{-1}\left(N_{\varepsilon / 2}\left(p_{i}\right)\right) \times N_{\varepsilon / 2}\left(p_{j}\right)$ a diagonal set if $i=j$. We prove that the $i^{t h}$ diagonal set intersects only the $(i-1)^{s t}$ and $(i+1)^{s t}$ diagonal sets, so that $W-U$ is exactly the union of nondiagonal sets, and hence open. Suppose

$$
\left\{f^{-1}\left(N_{\varepsilon / 2}\left(p_{i}\right)\right) \times N_{\varepsilon / 2}\left(p_{i}\right)\right\} \cap\left\{f^{-1}\left(N_{\varepsilon / 2}\left(p_{j}\right)\right) \times N_{\varepsilon / 2}\left(p_{k}\right)\right\} \neq \varnothing .
$$

Clearly, both $j$ and $k$ must assume values from $\{i-1, i, i+1\}$. Assume that $k=i+1$. By the definition of $W,|j-k|=3 n$, so that $j=i+1$ also. The argument for $j=i$ or $i-1$ is the same.

Theorem 6. Let $X$ be locally connected. Then $X$ has finitely many components if and only if $\tau_{u} \subset \tau$ for $C(X)$.

Proof. Suppose that $X$ is locally connected and has finitely many components. Then clearly the process used in Lemma 5 can be repeated a finite number of times to yield the desired result.

Now suppose that $X=\bigcup_{0}^{\infty} X_{i}$ where $X_{i}$, for $i>0$, is a component of $X$. Define $f\left(X_{i}\right)=i$ for every $i$. It is true that $V_{1 / 2}(f)$ is not open in $\tau$. In order to see this, choose $F_{U} \in \tau$ with $f \in F_{U}$ and $U=$ 
$\bigcup_{i \in \mathscr{A}} A_{i} \times B_{i}$. Then observe that $\left\{A_{i}: j \in B_{i}\right\}$ must cover $X_{j}$ and that for some $j \neq k,\left\{A_{i}: k \in B_{i}\right\}=\left\{A_{i}: j \in B_{i}\right\}$. If $g\left(X_{i}\right)=i$ for $i \neq j$ and $g\left(X_{j}\right)=k$, then $g \in F_{U}$, but $g \notin V_{1 / 2}(f)$.

Let $C^{*}(X)$ represent the collection of elements of $C(X)$ which are bounded. Our next lemma will enable us to show that when $X$ is locally connected, then $C^{*}(X)$ is closed in $C(X)$ with topology $\tau$ if and only if $\tau_{u} \subset \tau$.

Lemma 7. Let $X$ be locally connected. Then $C^{*}(X)$ is closed in $C(X)$ with topology $\tau$ if and only if $X$ has finitely many components.

Proof. Let $X$ be locally connected and suppose that $X$ has finitely many components, so that $\tau_{u} \subset \tau$. If $C^{*}(X)=C(X)$, then $C^{*}(X)$ is closed. If $C^{*}(X) \neq C(X)$, choose $f \in C(X)-C^{*}(X)$. Then $V_{1 / 2}(f) \in \tau$ and $V_{1 / 2}(f) \subset C(X)-C^{*}(X)$.

Suppose $X=\mathrm{U}_{0}^{\infty} X_{i}$ where $X_{i}$, for $i>0$, is a component and $X_{0}=X-\bigcup_{1}^{\infty} X_{i}$. Define $f\left(X_{i}\right)=i$. Then $f$ is unbounded, and we show that every $F_{U} \in \tau$ containing $f$ contains a bounded map. Let $F_{U} \in \tau$ where $U=\bigcup_{i \in \mathscr{A}} A_{i} \times B_{i}$ and $f \in F_{U}$. The set $\left\{A_{i}: j \in B_{i}\right\}$ covers $X_{j}$ and since there exists only a finite number of sets of the form $\left\{A_{i}: j \in B_{i}\right\}$, there exists an $M$ such that for any $n \geqq M$, $\left\{A_{i}: n \in B_{i}\right\}=\left\{A_{i}: j \in B_{i}\right\}$ for some $j<M$. Define $g$ such that $g\left(X_{i}\right)=i$ for $i \leqq M$, and for $n>M, g\left(X_{n}\right)=j$ where $j<M$ and found as indicated above. Then $g \in F_{U}$ while $g \in C^{*}(X)$.

Combining Lemma 7 with Theorem 6 we have the following result.

Theorem 8. Let $X$ be locally connected. Then $C^{*}(X)$ is closed in $C(X)$ with topology $\tau$ if and only if $\tau_{u} \subset \tau$.

We now turn to the question of necessary and sufficient conditions in order that $\tau \subset \tau_{u}$. Theorems 3 and 4 indicate that when $X$ is countably compact, then $\tau \subset \tau_{u}$ on $C(X)$. But the converse of this statement is not true in general, since $C(X)$ might consist of constant maps only and in that event $\tau=\tau_{u}$ on $C(X)$ regardless of the properties of $X$.

Before giving an answer to this question, we need a definition.

Definition 9. A completely regular space $X$ is well-separated if for any sequence, $\left\{x_{n}\right\}$, in $X$ having no cluster points, there exists a neighborhood-finite sequence of open sets, $\left\{U_{n}\right\}$, with $x_{n} \in U_{n}$ for each $n$. 
S. Naimpally and C. Pareek [4] show in an example that $\tau \not \subset \tau_{u}$ on $C(X)$ when $X=R$. We wish to show that under certain conditions, when $X$ is not countably compact, then $\tau \not \subset \tau_{u}$ on $C(X)$. When $X$ is not countably compact, we have the existence of a sequence of distinct points with no cluster points, say $\left\{x_{n}\right\}$. We wish to define a continuous function $f$ on this sequence such that $f\left(x_{n}\right)=1-1 / n$ and extend it to all of $X$ in such a way that $f(X) \subset[0,1)$. For this task, the condition of well-separatedness will be shown to be, in a sense, natural.

THEOREM 10. If $X$ is well-separated, then $\tau \subset \tau_{u}$ for $C(X)$ if and only if $X$ is countably compact.

Proof. Suppose that $X$ is not countably compact and that $\left\{x_{n}\right\}$ is a sequence in $X$ having no cluster points. We can take $\left\{x_{n}\right\}$ to consist of distinct points. There exists a neighborhood-finite sequence, $\left\{U_{n}\right\}$, of open sets with $x_{n} \in U_{n}$ for each $n$, and further we can require that $\bar{U}_{n} \cap \bar{U}_{m}=\varnothing$ for $n \neq m$. Choose $F_{V} \in \tau$ with $V=X \times$ $N_{1}(0)$. Define $f_{n}$ on $\bar{U}_{n}$ such that $f_{n}\left(x_{n}\right)=1-1 / n$ and $f_{n}\left(\bar{U}_{n}-U_{n}\right)=0$ for each $n>0$, and define $f_{0}$ on $c \bigcup_{1}^{\infty} U_{n}$ to be the zero map. Then there exists a unique continuous function $f$ which is an extension of each $f_{n}$. Clearly $f \in F_{V}$, but no $V_{\varepsilon}(f)$ is contained in $F_{V}$.

4. Properties of well-separatedness. In the proof of Theorem 10 we wished to extend a continuous function on a sequence having no cluster points to all of the space. We imposed the condition of well-separatedness. This condition is natural because of the following result. (A subspace $Y \subset X$ is $C$-embedded in $X$ if each element of $C(Y)$ is the restriction of an element of $C(X)$.)

THEOREm 11. A completely regular space $X$ is well-separated if and only if every sequence in $X$ having no cluster point is $C$-embedded in $X$.

Proof. Only half of the assertion needs comment. Let $\left\{x_{n}\right\}$ be a sequence of distinct points in $X$ having no cluster points, and define $f\left(x_{n}\right)=n$ for each $n$. Extend $f$ to a continuous function on all of $X$. Then $\left\{f^{-1}\left(N_{1 / 3}(n)\right)\right\}$ is the desired neighborhood-finite collection.

Dugundji [2] defines a completely regular space to be weakly normal if disjoint closed sets, one of which is countable, can be separated by disjoint open sets. Then he shows that in weakly normal spaces, countable compactness and pseudocompactness are equivalent. Even though the class of well-separated spaces is larger 
than the class of weakly normal spaces, countable compactness and pseudocompactness are equivalent there also.

Corollary 12. If $X$ is well-separated, then countable compactness and pseudocompactness are equivalent.

Proof. If $X$ is not countably compact, then there exists a sequence of distinct points, $\left\{x_{n}\right\}$, with no cluster points and if $f\left(x_{n}\right)=n$ for each $n$, then $f$ has a continuous extension to all of $X$ by Theorem 11 .

THEOREM 13. If $X$ is weakly normal, then $X$ is well-separated.

Proof. Let $\left\{x_{n}\right\}$ be a sequence in $X$ having no cluster points, and choose a sequence of open sets, $\left\{U_{n}\right\}$, with $x_{n} \in U_{n}$ for each $n$ and such that $U_{n} \cap U_{m}=\varnothing$ if $x_{n} \neq x_{m}$. Then $\cup\left\{x_{n}\right\}$ and $c \cup U_{n}$ are disjoint closed sets and there exist disjoint open sets $V$ and $W$ containing $\cup\left\{x_{n}\right\}$ and $c \cup U_{n}$ respectively. Define $V_{n}=V \cap U_{n}$ for each n. Then $\left\{V_{n}\right\}$ is the desired neighborhood-finite collection. For if $x \in U_{n}$ for some $n$, then $U_{n} \cap V_{m}=\varnothing$ for $x_{m} \neq x_{n}$. Further, if $x \notin U_{n}$ for every $n$, then $x \in c \cup U_{n}$ implying that $x \in W$ and $W \cap V_{n}=\varnothing$ for every $n$.

Theorem 13 shows that well-separatedness is strictly weaker than normality, since weak normality is strictly weaker than normality. In order to see that well-separatedness is strictly stronger than complete regularity, let $[0, \Omega]$ be the space of ordinals less than or equal to the first uncountable ordinal and $[0, \omega]$ be the space of ordinals less than or equal to the first countable ordinal. Then $[0, \Omega] \times[0, \omega]-\{(\Omega, \omega)\}$ is a completely regular space that is not well-separated. To see this, note that $\{(\Omega, n)\}$ has no cluster points. Choose $\left\{U_{n}\right\}$ with $(\Omega, n) \in U_{n}$ for each $n$. Then any sequence $\left\{z_{n}\right\}$ such that $z_{n} \in U_{n}$ for each $n$ and the first coordinate of $z_{n}$ differs from $\Omega$ can be seen to cluster. This shows that $\left\{U_{n}\right\}$ is not neighborhood-finite.

We list several more applications of the well-separated property.

THEOREM 14. Let $X$ be well-separated.

(a) If $X$ is not countably compact, then $C(X)$ with topology $\tau_{u}$ is not separable.

(b) The set of units in $C(X)$ with topology $\tau_{u}$ is open if and only if $X$ is countably compact.

(c) Scalar multiplication (or multiplication) is continuous in $C(X)$ with topology $\tau$ if and only if $X$ is countably compact. 
(d) The space $X$ is countably compact if and only if $f(X)$ is closed for any $f \in C(X)$.

Proof. (a) If $X$ is not countably compact, there exists a sequence, $\left\{x_{n}\right\}$, of distinct points with no cluster points. Let $\left\{f_{n}\right\}$ be a countable collection of functions. Define $g\left(x_{n}\right)=f_{n}\left(x_{n}\right)+1$ for each $n$ and then extend $g$ to a continuous function on all of $X$ by Theorem 11. Clearly, $V_{1 / 2}(g)$ contains no $f_{n}$.

(b) If $X$ is countably compact and $f \in C(X)$ such that $f>0$, then by Lemma 2, there exists an $\varepsilon>0$ such that $V_{\varepsilon}(f)$ contains only positive functions. If $X$ is not countably compact, choose a sequence, $\left\{x_{n}\right\}$ of distinct points having no cluster points and define $f\left(x_{n}\right)=1 / n$ for $n>0$, and then extend $f$ to a continuous function on $X$ in such a way that $f>0$. Then $f$ is a unit while for any $\varepsilon>0, V_{\varepsilon}(f)$ contains nonunits.

(c) If $X$ is countably compact, $\tau=\tau_{u}$ and multiplication, as well as scalar multiplication, is continuous in $C(X)$ with topology $\tau_{u}$. If $X$ is not countably compact, choose a sequence of distinct points $\left\{x_{n}\right\}$ and define $f\left(x_{n}\right)=n$. Extend $f$ to a continuous function on all of $X$. Let $g$ be the zero map. In order to see that scalar multiplication is not continuous, note that $0 \cdot f=g$, while $N_{1 / n}(0) \cdot f \not \subset F_{U}$ where $U=X \times N_{\varepsilon}(0)$. (We write $N_{1 / n}(0) \cdot f$ for $\left\{a \cdot f \mid a \in N_{1 / n}(0)\right\}$ ). The argument for multiplication is almost the same.

(d) If $X$ is not countably compact, then there exists a sequence of distinct points, $\left\{x_{n}\right\}$, which do not cluster. If $f$ is defined so that $f\left(x_{n}\right)=1 / n$ and then extended to a continuous function on $X$ in such a way that $f(X) \subset(0,1]$, then $f(X)$ is not closed. If $X$ is countably compact, then $f(X)$ is compact and thus closed.

\section{REFERENCES}

1. R. Arens and J. Dugundji, Topologies for function spaces, Pacific J. Math. 1 (1951), 5-31.

2. J. Dugundji, Topology, Allyn and Bacon, 1966.

3. S. Naimpally, Graph topology for function spaces, Trans. Amer. Math. So. 123 (1966), 267-72.

4. S. Naimpally and C. Pareek, Graph topologies for functions spaces II, (to appear in Prace Mat).

5. H. Poppe, Über Graphentopologien fïr Abbildungsräume, I, Bull. Acad. Pol. Sci. 15 (1967), 71-80.

Received June 4, 1969, and in revised form October 22, 1969. This work was part of a dissertation written under the direction of Professor Paul E. Long at the University of Arkansas while the author was the recepient of a NASA Traineeship. 


\section{PACIFIC JOURNAL OF MATHEMATICS}

\section{EDITORS}

\author{
H. SAMELSON \\ Stanford University \\ Stanford, California 94305

\section{Richard Pierce} \\ University of Washington \\ Seattle, Washington 98105
}

J. DugundJI

Department of Mathematics

University of Southern California

Los Angeles, California 90007

RichaRd ARENS

University of California

Los Angeles, California 90024

\section{ASSOCIATE EDITORS}
E. F. BECKENBACH
B. H. NeUMANN
F. WOLE
K. YoshidA

\section{SUPPORTING INSTITUTIONS}

\author{
UNIVERSITY OF BRITISH COLUMBIA \\ CALIFORNIA INSTITUTE OF TECHNOLOGY \\ UNIVERSITY OF CALIFORNIA \\ MONTANA STATE UNIVERSITY \\ UNIVERSITY OF NEVADA \\ NEW MEXICO STATE UNIVERSITY \\ OREGON STATE UNIVERSITY \\ UNIVERSITY OF OREGON \\ OSAKA UNIVERSITY \\ UNIVERSITY OF SOUTHERN CALIFORNIA
}

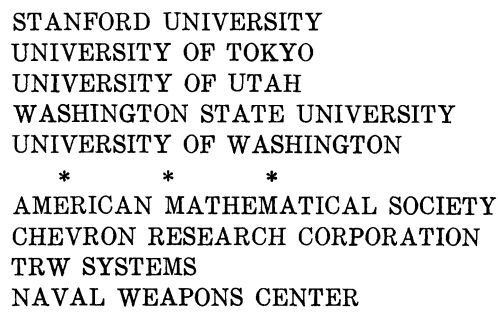

The Supporting Institutions listed above contribute to the cost of publication of this Journal, but they are not owners or publishers and have no responsibility for its content or policies.

Mathematical papers intended for publication in the Pacific Journal of Mathematics should be in typed form or offset-reproduced, (not dittoed), double spaced with large margins. Underline Greek letters in red, German in green, and script in blue. The first paragraph or two must be capable of being used separately as a synopsis of the entire paper. The editorial "we" must not be used in the synopsis, and items of the bibliography should not be cited there unless absolutely necessary, in which case they must be identified by author and Journal, rather than by item number. Manuscripts, in duplicate if possible, may be sent to any one of the four editors. Please classify according to the scheme of Math. Rev. Index to Vol. 39. All other communications to the editors should be addressed to the managing editor, Richard Arens, University of California, Los Angeles, California, 90024.

50 reprints are provided free for each article; additional copies may be obtained at cost in multiples of 50 .

The Pacific Journal of Mathematics is published monthly. Effective with Volume 16 the price per volume (3 numbers) is $\$ 8.00$; single issues, $\$ 3.00$. Special price for current issues to individual faculty members of supporting institutions and to individual members of the American Mathematical Society: $\$ 4.00$ per volume; single issues $\$ 1.50$. Back numbers are available.

Subscriptions, orders for back numbers, and changes of address should be sent to Pacific Journal of Mathematics, 103 Highland Boulevard, Berkeley, California, 94708.

PUBLISHED BY PACIFIC JOURNAL OF MATHEMATICS, A NON-PROFIT CORPORATION

Printed at Kokusai Bunken Insatsusha (International Academic Printing Co., Ltd.), 7-17, Fuj̣imi 2-chome, Chiyoda-ku, Tokyo, Japan. 


\section{Pacific Journal of Mathematics}

\section{Vol. 35, No. $2 \quad$ October, 1970}

Valentin Danilovich Belousov and Palaniappan L. Kannappan, Generalized Bol functional equation .................................... 259

Charles Morgan Biles, Gelfand and Wallman-type compactifications ........... 267

Louis Harvey Blake, A generalization of martingales and two consequent convergence theorems .................................... 279

Dennis K. Burke, On p-spaces and $w \Delta$-spaces..................... 285

John Ben Butler, Jr., Almost smooth perturbations of self-adjoint operators . . . . . . 297

Michael James Cambern, Isomorphisms of $C_{0}(Y)$ onto $C(X) \ldots \ldots \ldots \ldots \ldots . \ldots 307$

David Edwin Cook, A conditionally compact point set with noncompact closure ... 313

Timothy Edwin Cramer, Countable Boolean algebras as subalgebras and homomorphs .........................................

John R. Edwards and Stanley G. Wayment, A v-integral representation for linear operators on spaces of continuous functions with values in topological vector spaces.............................................

Mary Rodriguez Embry, Similarities involving normal operators on Hilbert

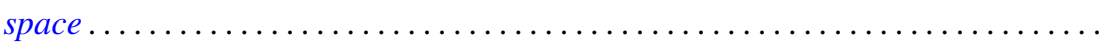

Lynn Harry Erbe, Oscillation theorems for second order linear differential

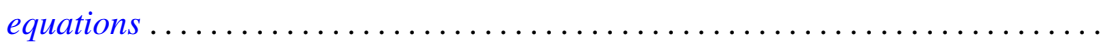

William James Firey, Local behaviour of area functions of convex bodies .......... Joe Wayne Fisher, The primary decomposition theory for modules ..............

Gerald Seymour Garfinkel, Generic splitting algebras for Pic ..................

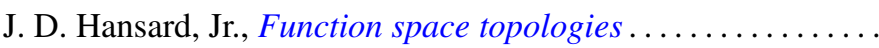

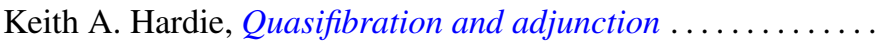

G. Hochschild, Coverings of pro-affine algebraic groups ...........

Gerald L. Itzkowitz, On nets of contractive maps in uniform spaces ..

381

389

399

417

Melven Robert Krom and Myren Laurance Krom, Groups with free nonabelian subgroups....................................

James Robert Kuttler, Upper and lower bounds for eigenvalues by finite differences ......................................

Dany Leviatan, A new approach to representation theory for convolution transforms . . .

Richard Beech Mansfield, Perfect subsets of definable sets of real numbers ...

Brenda MacGibbon, A necessary and sufficient condition for the embedding of a

Lindelof space in a Hausdorff $\mathscr{H} \sigma$ space ..................

David G. Mead and B. D. McLemore, Ritt's question on the Wronskian ....

Edward Yoshio Mikami, Focal points in a control problem .....

Paul G. Miller, Characterizing the distributions of three independent n-dimensional random variables, $X_{1}, X_{2}, X_{3}$, having analytic characteristic functions by the joint distribution of $\left(X_{1}+X_{3}, X_{2}+X_{3}\right)$. . .

P. Rosenthal, On the Bergman integral operator for an elliptic partial differential equation with a singular coefficient....

Douglas B. Smith, On the number of finitely generated $O$-group 